\title{
Research Article \\ Frequency of intradialytic hypotensive episodes: old problem, new insights
}

\author{
Ana Rocha, MD ${ }^{\mathrm{a}, \mathrm{b}, *}$, Clemente Sousa, $\mathrm{PhD}^{\mathrm{a}, \mathrm{c}}$, Paulo Teles, $\mathrm{PhD}^{\mathrm{d}}$, Augusto Coelho, $\mathrm{MD}^{\mathrm{a}}$, and \\ Eva Xavier, MD \\ ${ }^{a}$ São Francisco Dialysis Unit, Porto, Portugal; \\ ${ }^{b}$ Unit for Multidisciplinary Research in Biomedicine-UMIB, Instituto de Ciências Biomédicas Abel Salazar-ICBAS, University of Porto, \\ Porto, Portugal; \\ ${ }^{c}$ Nursing School of Porto, Porto, Portugal; and \\ ${ }^{d}$ School of Economics and LIAAD-INESC Porto LA, Porto University, Porto, Portugal
}

Manuscript received May 26, 2015 and accepted July 18, 2015

\begin{abstract}
Symptomatic intradialytic hypotension (IDH) continues to be an important complication of hemodialysis treatment. There is some evidence that besides an IDH episode, repeated episodes could represent an even more important independent risk factor for mortality in hemodialysis patients. A retrospective cross-sectional study was performed to study 18 dialysis treatments in 43 patients during 6 weeks. Relationships of IDH episodes with baseline variables were examined using a Poisson regression model (generalized linear model). IDH was frequent (93\% of patients) and highly variable by patient $(0 \%-100 \%)$. Multivariate analysis showed that patients who experienced frequent hypotensive episodes had a lower dry weight ( $90 \%$ confidence interval [CI]: 0.95-0.99), higher phosphorus levels (90\% CI: 1.07-1.47), greater prevalence of diabetes mellitus (90\% CI: 1.11-2.71), and hypertension (90\% CI: 1.04-2.45). Dry weight, hypertension, and phosphorus levels are modifiable risk factors to possibly reduce the rate of IDH episodes. The potential protective role of phosphorus warrants further investigation. J Am Soc Hypertens 2015;9(10):763-768. @ 2015 American Society of Hypertension. All rights reserved.
\end{abstract}

Keywords: Diabetes; hemodialysis; hypotension; phosphorus.

\section{Introduction}

Intradialytic hypotension (IDH) is a common event that occurs during the hemodialysis procedure. It has three essential components: a drop in blood pressure (BP) generally defined as $\geq 20 \mathrm{~mm} \mathrm{Hg}$ systolic BP or $\geq 10 \mathrm{~mm} \mathrm{Hg}$ in mean arterial pressure; the presence of symptoms of end-organ ischemia; and an intervention carried out by the dialysis staff. ${ }^{1}$ On the basis of the National Kidney Foundation Kidney Disease Outcomes Quality Initiative definition, IDH occurs in approximately $20 \%-30 \%$ of all hemodialysis sessions.

Factors such as rapid fluid removal through ultrafiltration; abrupt changes in serum osmolar, acid-base, and

Conflict of interest: None.

*Corresponding author: Ana Rocha, MD, São Francisco Dialysis Unit, Rua da Bolsa ${ }^{\circ}$ 87, 4050-116 Porto, Portugal. Tel: 222062 100; Fax: 222031150.

E-mail: acrisbraga@gmail.com electrolyte composition; activation of neurohormonal axes; and dialytic removal of vasoactive medications superimposed on a stiff vasculature and uremic inflammation suggest that BP during hemodialysis treatments may be exceptionally labile; this is consistent with clinical experience. In turn, rapid downward BP fluctuations may lead to repetitive episodes of overt or subclinical end-organ ischemic injury, and abrupt upward fluctuations may lead to increased capillary endothelial shear stress damage ${ }^{2,3}$

A growing body of evidence suggests that BP variability portends worse outcomes in both the general and kidney disease populations. ${ }^{2,4,5}$ A large series evaluating the association between IDH and outcomes for cardiovascular events and mortality revealed that IDH was associated with all-cause mortality, myocardial infarction, hospitalization for heart failure/volume overload or cardiovascular mortality, and major cardiac events. ${ }^{6}$ In a cohort of 113,255 hemodialysis patients, U-shaped associations were found between change in BP during dialytic treatment 
and mortality independent of prehemodialysis BP levels. ${ }^{5}$ Large decreases in systolic and diastolic BP during session (less than -30 and less than $-15 \mathrm{~mm} \mathrm{Hg}$, respectively) were associated with increased mortality, whereas more moderate reductions (delta systolic BP of $-14 \mathrm{~mm} \mathrm{Hg}$ and a delta diastolic BP of $-6 \mathrm{~mm} \mathrm{Hg}$, respectively) were associated with the greatest survival. ${ }^{5}$

Although BP lability during hemodialysis has long been recognized, little is known about factors that promote systematic IDH episodes. Our study has been designed to evaluate the frequency of symptomatic IDH and to uncover the variables associated with those systematic episodes.

\section{Methods}

\section{Study Design}

The criteria to be eligible for the study were participants with $>18$ years of age, medically stable, undergoing hemodialysis three times per week for a minimum of 4 hours per session and for more than 2 months. Because BP immediately after dialysis therapy initiation may fluctuate more widely due to dry weight probing and medication titration, we excluded patients on hemodialysis therapy for less than 2 months.

Patients entered the cohort between September 12, 2012, and October 22, 2012. In total, the analytical cohort consisted of 43 unique patients.

Laboratory parameters were measured monthly according to the organization protocol; all processing was conducted at a single clinical laboratory.

Dialytic session data were recorded on a session-to-session basis. Patients were dialyzed on either a MondayWednesday-Friday or Tuesday-Thursday-Saturday schedule.

$\mathrm{BP}$ was measured with the patient in the seated position using automated oscillometric devices immediately before, after, and during all treatment sessions.

Target weight was evaluated clinically (peripheral edema, signs of pulmonary congestion, intradialytic and extradialytic BP course, and muscle cramps) and by (changes in) the cardiopulmonary radiologic aspect. Excess weight at the start of dialysis was defined as the difference between predialysis weight and target weight. The prescribed ultrafiltration volume was calculated by adding the estimated intradialytic fluid intake (usually $500-750 \mathrm{~mL}$ ) to the excess weight. However, for all analyses, the exact ultrafiltration volume as delivered by the dialysis apparatus was used.

Hypertension was defined as BP $>140 \mathrm{~mm} \mathrm{Hg}$ systolic or $>90 \mathrm{~mm} \mathrm{Hg}$ diastolic. Heart failure was defined as left ventricular ejection fraction $<50 \%$.

\section{Dialysis Settings}

All patients were dialyzed with synthetic hollow-fiber dialyzers, Elisio ${ }^{-15} \mathrm{H}, 17 \mathrm{H}$, or $21 \mathrm{H}$ (Nipro). All the sessions consisted of standard hemodialysis using standard dialysis solutions. Standard bicarbonate concentrations were adjusted if necessary $(30-35 \mathrm{mmol} / \mathrm{L})$. Two needles were used in all patients with an arteriovenous access. Blood flow ranged from 300 to $450 \mathrm{~mL} / \mathrm{min}$. Dialysate flow was 500 or $700 \mathrm{~mL} / \mathrm{min}$. Blood flow and dialysate flow were kept constant throughout the study period in individual patients. All patients were dialyzed with a constant ultrafiltration rate. Dialysate temperature was $35.5^{\circ} \mathrm{C}$ or $36.0^{\circ} \mathrm{C}$ and was kept constant during the study period for individual patients. Patients received a light meal and two cups of coffee or tea during treatment.

Individual treatment characteristics were not changed in the course of the study.

\section{Data Collection and Description}

All data were obtained from the clinical electronic medical record.

Demographic characteristics (age, gender, dialysis vintage, and vascular access type) and comorbid conditions (diabetes, hypertension, and heart failure) were considered as of cohort entry. Laboratory values (parathyroid hormone, phosphorus, calcium, and hemoglobin) and dialytic session characteristics (predialysis weight, postdialysis weight, and interdialytic weight gain; body mass index; and erythropoietin dose used) were considered as the mean value measured during the exposure period. Prescribed antihypertensive medications were also considered, and it was not changed for the purposes of the study.

The number of IDH episodes in 18 consecutive hemodialysis sessions was recorded for each patient. The responsive measures included saline administration or premature cessation of dialysis.

\section{Statistical Analysis}

Quantitative variables were described by the mean and the standard deviation (SD). The distribution of categorical variables was represented by proportions.

A Poisson regression model was used. The independent variables considered were gender, age, dialysis vintage, diabetes, hypertension, heart failure, number of antihypertensive drugs taken by the patient, hemoglobin, parathyroid hormone, calcium and phosphorus level, ultrafiltration volume, dry weight, body mass index, and erythropoietin dose.

The model considered first included all the variables and those whose estimated parameters were not significant at the $10 \%$ level were removed, which leaded to a fitted model including the presence of diabetes, hypertension, the phosphorus level, and dry weight.

The regression model was fitted with overdispersion adjustment and the estimation results of the final model included parameter estimates, $P$-values, $90 \%$ confidence intervals (CIs), incidence rate ratios (the exponentials of the model parameters), and their $90 \%$ CIs. To test the overall significance 
of the fitted model, the chi-square deviance goodness-of-fit statistic was 37.7 ( 4 degrees of freedom), with a $P$-value of approximately 0 , showing that the model was significant.

\section{Results}

\section{Cohort and Treatment Characteristics}

Overall, 43 Caucasian patients underwent a total of 18 dialysis treatments, during which three qualifying BP measurements were recorded.

Mean age was 66.6 years (SD, 13.2), most ages range between 60 and 80 years, with only a few lower than 50 years. Mean dialysis vintage was 86 months, with a range of 2-388 months (SD, 102.2). All patients were dialyzed by fistula, $58.1 \%$ were men, $27.9 \%$ were diabetic, and $65.1 \%$ were prescribed antihypertensive medications with a maximum of four drugs. Patients taking one $(32 \%)$ or two drugs $(39 \%)$ were the large majority. Near half of the patients were under B-blocker (48.8\%), a quarter used angiotensin receptor blockers $(25.6 \%), 23.3 \%$ used a calcium channel blocker, and $18.6 \%$ angiotensin-converting enzyme inhibitor; only a small number of patients (14\%) were under other antihypertensive agents.

Heart failure was present in $23.3 \%$ of patients.

Concerning laboratory measures of the study population, the mean values of hemoglobin, parathyroid hormone, calcium, and phosphorus were $11.5 \pm 0.6 \mathrm{~g} / \mathrm{dL}, 304 \pm 154 \mathrm{pg} /$ $\mathrm{mL}, 8.8 \pm 0.4 \mathrm{mg} / \mathrm{dL}$, and $4.8 \pm 1.2 \mathrm{mg} / \mathrm{dL}$, respectively. Mean erythropoietin dose was $105.4 \mathrm{U} / \mathrm{kg} /$ week (SD, 23.8).
The mean ultrafiltration volume was $2.7 \pm 0.8 \mathrm{~L}$ (range, $0.2-4.9 \mathrm{~L}$ ) with a mean dry weight of $62.4 \pm 9.9 \mathrm{~kg}$ (range, 44.5-88.8 kg) and a mean body mass index of $23.5 \pm 3.8 \mathrm{~kg} / \mathrm{m}^{2}$ (range, $16.3-31.4 \mathrm{~kg} / \mathrm{m}^{2}$ ). Mean predialysis and postdialysis weights were 64.9 and $62.4 \mathrm{~kg}$, respectively.

\section{BP Metric}

A description of BP parameters across the cohort is provided in Figures 1 and 2 .

IDH was frequent, present in $30.7 \%$ of all treatments and $93 \%$ of all patients, and was highly variable by patient (between one and all treatments).

The large majority $(76.7 \%$ ) had seven or less IDH episodes, and few patients had more than $10 \mathrm{IDH}$ events, with a maximum number of 19 . The most frequent number of IDH episodes were 2 and 3 (14\% each), 4 (11.6\%), and 6 $(9.3 \%)$, and the mean and the median were 5.5 and 4 events, respectively. The mean predialysis and postdialysis BP were $140.4 \pm 25.6 \mathrm{~mm} \mathrm{Hg}$ (range, 63.9-213.7 mm Hg) and $141.2 \pm 23.8 \mathrm{~mm} \mathrm{Hg}$ (range, 68.9-184.4 $\mathrm{mm} \mathrm{Hg}$ ), respectively.

In multivariate analysis (Table 1), independent risk factors for recurrent IDH episodes included diabetes mellitus (odds ratio [OR], 1.75; 90\% CI: 1.11-2.71), higher phosphorus levels (OR, 1.26; 90\% CI: 1.07-1.47), hypertension (OR, 1.58; 90\% CI: 1.04-2.45), and lower dry weight (OR, 0.97; 90\% CI: 0.95-0.99).

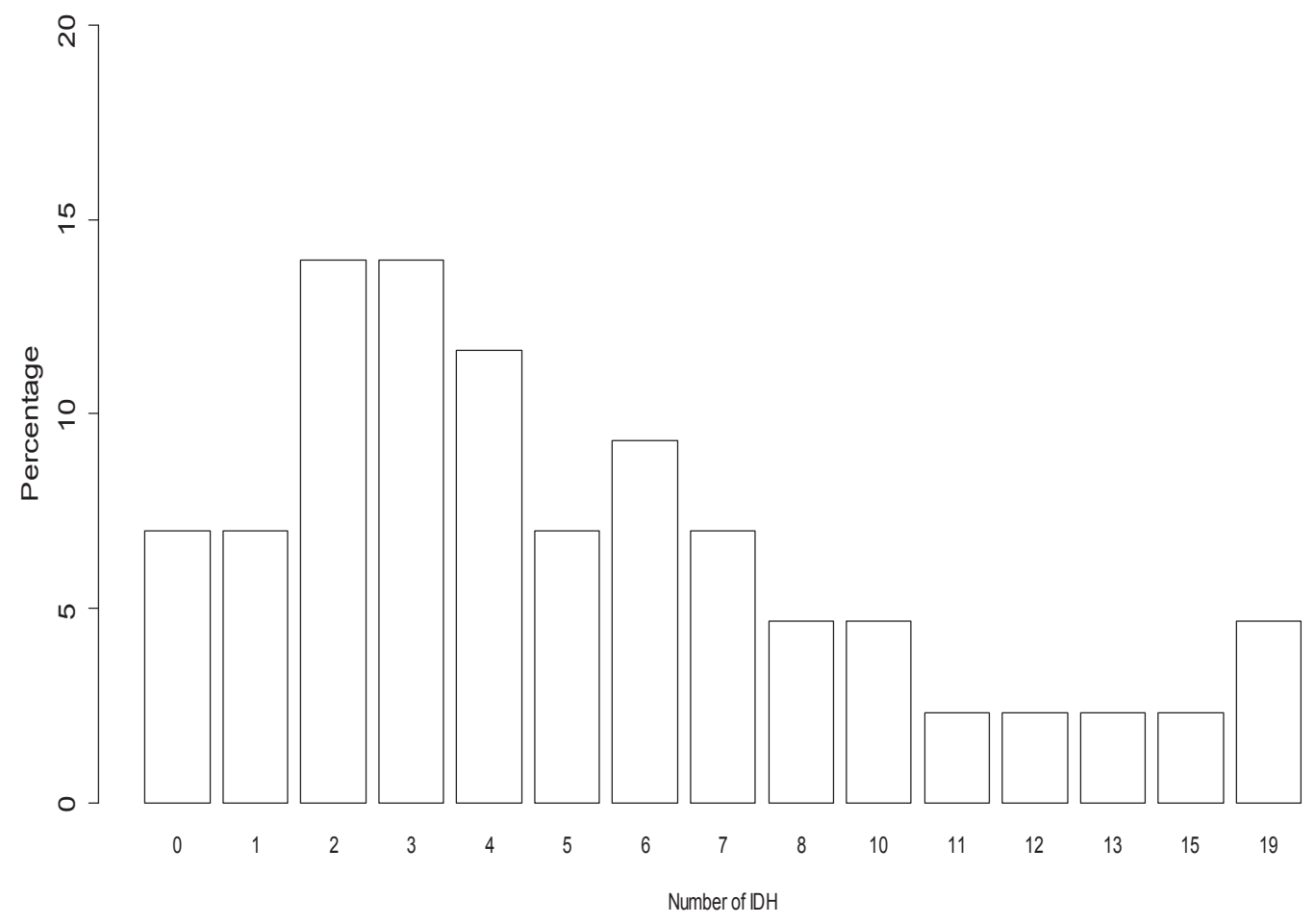

Figure 1. Distribution of the number of IDH. IDH, intradialytic hypotension. 


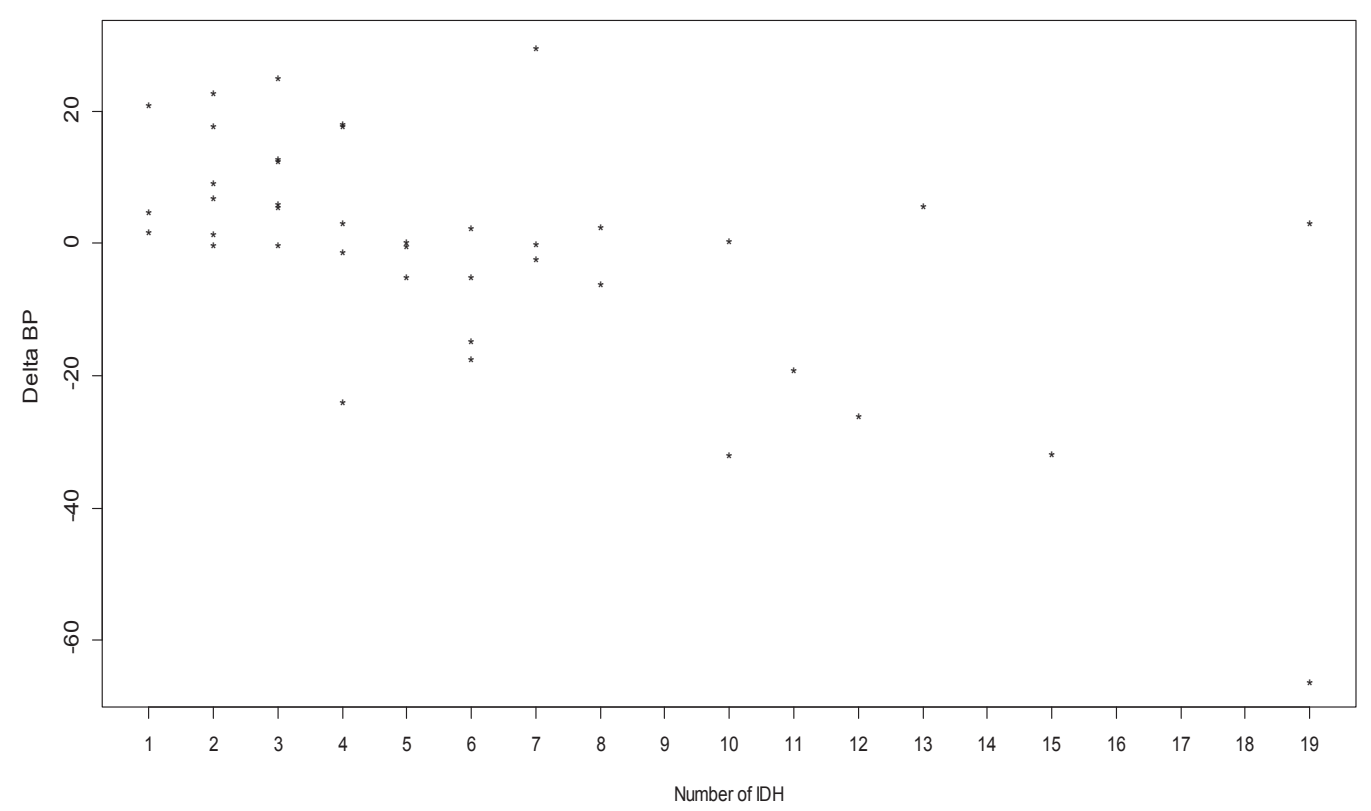

Figure 2. Delta systolic blood pressure in IDH episodes. BP, blood pressure; IDH, intradialytic hypotension.

\section{Discussion}

The effect of frequent or occasional dialysis-associated hypotension on survival of patients on maintenance hemodialysis was previously studied, revealing that mortality in patients with frequent episodes is significantly higher than in those without such events; the authors suggest that the high mortality observed in patients with frequent IDH may be considered as a warning sign for patients being at high risk of death. ${ }^{7}$

Another study evaluating frequency of IDH concluded that identifying practice patterns associated with IDH coupled with routine reporting, it will facilitate medical management and may result in the prevention of IDH, decreased mortality, and decreased hospitalization. ${ }^{8}$

IDH episodes were present in $30.7 \%$ of all treatments in a sample size of 43 patients with 774 treatments. This frequency is consistent with previous reports. ${ }^{4,6,7}$ We also verified that most patients (93\%) had IDH, Sands et $\mathrm{al}^{8}$ refer a rate of $75.1 \%$ and in spite of the wide variation of its definition in the literature, and such numbers emphasize the clinical significance of the problem of IDH.

In this population-based cohort study, we found several independent risk factors associated with recurrent IDH episodes. We conclude from the fitted model that the number of IDH episodes is positively affected by being diabetic, having high $\mathrm{BP}$ and by the phosphorus level and is negatively affected by the dry weight.

Extracellular volume is a major determinant of BP in dialysis patients. ${ }^{9}$ Ultrafiltration during dialysis leads to a fall in the patient's blood volume. ${ }^{10}$ Greater fluid removal (whether considered as volume or rate either absolute or relative to total body water) was associated with greater BP variability in other studies. ${ }^{3}$ Intradialytic hypotension is thought to occur most often when the rate of fluid removal exceeds the rate of refill from the interstitial space into the vasculature. In our report, we found a significant association between recurrent IDH episodes and lower dry weight although no difference has been noted for ultrafiltration rate. This raises the hypothesis that with the same ultrafiltration rate, patients

\section{Table 1}

Poisson regression estimation results

\begin{tabular}{|c|c|c|c|c|c|}
\hline \multirow[t]{2}{*}{ Variable } & \multicolumn{3}{|c|}{ Estimated Parameters } & \multicolumn{2}{|c|}{ Incidence Rate Ratios } \\
\hline & Estimate & $P$-Value & $90 \% \mathrm{CI}$ & Ratio & $90 \% \mathrm{CI}$ \\
\hline Intercept & 1.6967 & .0517 & $0.3291,3.1127$ & 5.4561 & $1.3897,2.4808$ \\
\hline Hypertension & 0.4545 & .0861 & $0.0432,0.8953$ & 1.5754 & $1.0442,2.4480$ \\
\hline Phosphorus level & 0.2283 & .0244 & $0.0665,0.3873$ & 1.2564 & $1.0687,1.4730$ \\
\hline Dry weight & -0.0258 & .0462 & $-0.0465,-0.0053$ & 0.9746 & $0.9546,0.9947$ \\
\hline
\end{tabular}

CI, confidence interval. 
with lower dry weight become more symptomatic that patients with a higher one. This could be due to a dehydration state in these patients with predisposition to hypotension. ${ }^{11}$ Another possible explanation is related to the percentage of weight variation; for the same ultrafiltration rate, it is higher in a patient with lower dry weight. In our cohort for each kilogram increase in the dry weight, the average number of IDH episodes decreased $2.5 \%$.

An interesting finding was that patients with high BP had a much higher hypotension rate, showing that patients with hypertension can have up to 2.4 times more IDH episodes on average, independent of use of antihypertensive agents, holding constant the dry weight. Although prehemodialysis BP measurements may demonstrate greater variability and may not consistently correlate with interdialytic ambulatory BP monitoring, their use is supported by the Kidney Disease Outcomes Quality Initiative guidelines and has greater applicability to clinical practice.

Our conclusion is in opposition with a study by Davenport et $\mathrm{al}^{12}$ : This audited BP control and symptomatic IDH requiring fluid resuscitation in the Greater London area renal centers, concluding that symptomatic IDH was more common in patients with lower predialysis BP and pulse pressures. Another study designed to evaluate if predialysis hypertension can prevent IDH revealed no correlation between the two variables. ${ }^{13}$

Hypertension is causally linked with heart failure and vascular calcification. ${ }^{12}$ So, one of the factors that could explain IDH in these patients, although not directly evaluated in our group, is cardiac dysfunction. A previous study of 172 patients found that those who experienced the hypotensive episodes had a greater prevalence of previous heart failure, systolic dysfunction, and left atrial volume index. ${ }^{14}$ Another reason could be associated with accelerated aging of arterial system with extensive calcification and stiffening of arterial walls. More recently, emerging evidence indicates that endothelial dysfunction may play a key role in hemodynamic instability during hemodialysis. ${ }^{15,16}$

Our results are in accordance with a study comparing 658 diabetic patients with 1535 nondiabetic individuals revealing that diabetic hemodialysis patients had higher BP, both before and after dialysis, associated with greater interdialytic weight gains and more frequent IDH, ${ }^{17}$ highlighting the role of higher BP and diabetes in IDH episodes. We found that diabetic patients had on average 1.75 times more IDH events that the nondiabetic. The presence of diabetes has great impact on the cardiovascular autonomic regulation during hemodialysis with an overall reduced autonomic activity and a blunted autonomic response. ${ }^{18}$ But there are a number of other reasons for higher susceptibility to hypotension in diabetic patients, including vascular damage, impaired left ventricular compliance, and the susceptibility to overhydration in the interdialytic interval. ${ }^{19}$

An interesting finding was the condition exhibiting the strongest association with a $50 \%$ maximum increase in the average number of IDH episodes for each $\mathrm{mg} / \mathrm{dL}$ increase in the phosphorus level.

Metabolic bone disease indexes and treatments that increase vascular stiffness would be associated with greater BP variability. Hemodialysis patients with severe secondary hyperparathyroidism are more likely to achieve normotensive and euvolemic status after parathyroidectomy, probably through improved heart function and reduced IDH episodes. ${ }^{20}$ In other contemporary cohort of 11,291 incident hemodialysis patients from a single dialysis organization, the authors demonstrated an association between greater predialysis systolic BP variation and higher calcium-phosphate product levels, ${ }^{21}$ probably associated with vascular calcification.

Other studies revealed that greater serum calcium level was associated inversely with BP variability; the same was not true for serum phosphorus level. ${ }^{3}$ Studies of the association between coronary artery calcification and phosphorus metabolism in uremic patients have been contradictory, but recently, this relationship has been proved. ${ }^{22,23}$ Control of phosphorus levels can potentially regulate arterial pressure.

The patient age, gender, dialysis vintage, heart failure, use of antihypertensive drugs and parathyroid hormone, calcium and hemoglobin level, body mass index, and erythropoietin dose were not associated with BP variability. This may be explained by the fact that some of the parameters were strictly controlled in a relatively narrow range among subjects in the present study.

The current work was a single-center cross-sectional design which potentially could affect the generality of the study and was not able to clarify whether these associations were causal. To apply the results to a general population on hemodialysis, we would have to include more patients in the future and additional prospective studies are needed to assess those associations.

\section{Conclusion}

We conclude from the fitted model that the number of IDH episodes was positively affected by being diabetic, having high BP and high phosphorus level and was negatively affected by dry weight. These were the only variables that significantly affected the number of IDH episodes among all the independent variables considered in the analysis. Strategies to improve phosphorus in diabetic and hypertensive patients should be further explored in a prospective controlled trial in an effort to verify causality with IDH episodes and possibly reduce its incidence.

\section{References}

1. Reilly RF. Attending rounds: a patient with intradialytic hypotension. CJASN 2014;9(4):798-803.

2. Flythe JE, Inrig JK, Shafi T, Chang TI, Cape K, Dinesh $\mathrm{K}$, et al. Association of Intradialytic blood 
pressure variability with increased all-cause and cardiovascular mortality in patients treated with long-term hemodialysis. Am J Kidney Dis 2013;61(6):966-74.

3. Flythe JE, Kunaparaju S, Dinesh K, Cape K, Feldman HI, Brunelli SM. Factors associated with intradialytic systolic blood pressure variability. Am J Kidney Dis 2012;59(3):409-18.

4. Flythe JE, Brubelli SM. Blood pressure variability and dialysis: variability may not always be the spice of life. J Am Soc Nephrol 2014;25:650-3.

5. Park J, Rhee CM, Sim JJ, Kim YL, Ricks J, Streja E, et al. A comparative effectiveness research study of the change in blood pressure during hemodialysis treatment and survival. Kidney Int 2013;84:795-802.

6. Stefánsson BV, Brunelli SM, Cabrera C, Rosenbaum D, Anum E, Ramakrishnan K, et al. Intradialytic hypotension and risk of cardiovascular disease. Clin J Am Soc Nephrol 2014;9(12):2124-32.

7. Tislér A, Akócsi K, Borbás B, Fazakas L, Ferenczi S, Görögh S, et al. The effect of frequent or occasional dialysis-associated hypotension on survival of patients on maintenance haemodialysis. Nephrol Dial Transplant 2003;18(12):2601-5.

8. Sands JJ, Usvyat LA, Sullivan T, Segal JH, Zabetakis P, Kotanko P, et al. Intradialytic hypotension: frequency, sources of variation and correlation with clinical outcome. Hemodial Int 2014;18(2):415-22.

9. Kuipers J, Usvyat LA, Oosterhuis JK, Dasselaar JJ, de Jong PE, Westerhuis R, et al. Variability of predialytic, intradialytic, and postdialytic blood pressures in the course of a week: a study of Dutch and US maintenance hemodialysis patients. Am J Kidney Dis 2013;62(4):779-88.

10. Leung KC, Quinn RR, Ravani P, MacRae JM. Ultrafiltration biofeedback guided by blood volume monitoring to reduce intradialytic hypotensive episodes in hemodialysis: study protocol for a randomized controlled trial. Trials 2014;15:483.

11. Agarwal R, Alborzi P, Satyan S, Light RP. Dry-weight reduction in hypertensive hemodialysis patients (DRIP): a randomized, controlled trial. Hypertension 2009;53(3):500-7.

12. Davenport A, Cox C, Thuraisingham R. Achieving blood pressure targets during dialysis improves control but increases intradialytic hypotension. Kidney Int 2008;73(6):759-64.
13. Takeda A, Toda T, Fujii T, Sasaki S, Matsui N. Can predialysis hypertension prevent intradialytic hypotension in hemodialysis patients? Nephron Clin Pract 2006; 103(4):c137-1743.

14. Barberato SH, Misocami M, Pecoits-Filho R. Association between left atrium enlargement and intradialytic hypotension: role of diastolic dysfunction in the hemodynamic complications during hemodialysis. Echocardiography 2009;26(7):767-71.

15. London G, Marchais S, Guérin A, Métivier F. [Arterial hypertension, chronic renal insufficiency and dialysis]. Nephrol Ther 2007;3(Suppl 3):S156-61.

16. Dubin R, Owens C, Gasper W, Ganz P, Johansen K. Associations of endothelial dysfunction and arterial stiffness with intradialytic hypotension and hypertension. Hemodial Int 2011;15(3):350-8.

17. Davenport A, Cox C, Thuraisingham R. Blood pressure control and symptomatic intradialytic hypotension in diabetic haemodialysis patients: a cross-sectional survey. Nephron Clin Pract 2008;109(2):c65-71.

18. Zitt E, Neyer U, Meusburger E, Tiefenthaler M, Kotanko P, Mayer G, et al. Effect of dialysate temperature and diabetes on autonomic cardiovascular regulation during hemodialysis. Kidney Blood Press Res 2008;31(4):217-25.

19. Locatelli F, Pozzoni P, Del Vecchio L. Renal replacement therapy in patients with diabetes and end-stage renal disease. J Am Soc Nephrol 2004;15(Suppl 1):S25-9.

20. Shih CJ, Tarng DC, Yang WC, Yang CY. Parathyroidectomy reduces intradialytic hypotension in hemodialysis patients with secondary hyperparathyroidism. Kidney Blood Press Res 2013;37(4-5):323-31.

21. Shafi T, Sozio SM, Bandeen-Roche KJ, Ephraim PL, Luly JR, St Peter WL, et al. Predialysis systolic BP variability and outcomes in hemodialysis patients. J Am Soc Nephrol 2014;25(4):799-809.

22. Wang M, Li H, You L, Yu X, Zhang M, Zhu R, et al. Association of serum phosphorus variability with coronary artery calcification among hemodialysis patients. PLoS One 2014;9(4):e93360.

23. Chang JF, Feng YF, Peng YS, Hsu SP, Pai MF, Chen HY, et al. Combined alkaline phosphatase and phosphorus levels as a predictor of mortality in maintenance hemodialysis patients. Medicine (Baltimore) 2014;93(18):e106. 